University of Nebraska - Lincoln

DigitalCommons@University of Nebraska - Lincoln

$9-18-2008$

Description of versatile optical polarimetric scatterometer that measures all 16 elements of the Mueller matrix for reflection and transmission: application to measurements of scatter cross sections, ellipsometric parameters, optical activity, and the complex chiral parameters

Ezekiel Bahar

Robert D. Kubik

Follow this and additional works at: https://digitalcommons.unl.edu/electricalengineeringfacpub

Part of the Computer Engineering Commons, and the Electrical and Computer Engineering Commons

This Article is brought to you for free and open access by the Electrical \& Computer Engineering, Department of at DigitalCommons@University of Nebraska - Lincoln. It has been accepted for inclusion in Faculty Publications from the Department of Electrical and Computer Engineering by an authorized administrator of DigitalCommons@University of Nebraska - Lincoln. 


\section{Description of versatile optical polarimetric scatterometer that measures all 16 elements of the Mueller matrix for reflection and transmission: application to measurements of scatter cross sections, ellipsometric parameters, optical activity, and the complex chiral parameters}

\author{
Ezekiel Bahar \\ University of Nebraska-Lincoln \\ Electrical Engineering Department \\ 201N WSEC \\ Lincoln, Nebraska 68588-0511 \\ E-mail: ebahar@unl.edu
}

Robert D. Kubik

Motorola, Inc.

1455 Pennsylvania Ave., NW Suite 900

Washington, DC 20005

\begin{abstract}
A detailed description of an optical polarimetric scatterometer, its capabilities, and special application are presented. This instrument measures the $4 \times 4$ Mueller matrix elements as well as bidirectional reflective distribution functions (scatter cross sections) for light that is scattered from a target. Incident polarized light at wavelengths $\lambda=0.6328$ and $\lambda=1.06 \mu \mathrm{m}$ can be directed toward the target in an arbitrary direction. The receiver can be located anywhere in the solid angle $4 \pi$. Backscatter measurements, most commonly used in remote sensing, can also be obtained. The scatterometer has been used to validate different analytical/numerical solutions to a broad class of electromagnetic scattering problems, in controlled laboratory experiments. The optical constants of liquids and solids can be determined by relating the Mueller matrix elements to the ellipsometric parameters. Recently, the relationships between the Mueller matrix elements and the parameters related to the optical activity have been derived. Thus, the scatterometer has the potential to detect, identify, and characterize optical rotation and circular dichroism of biological and chemical materials that possess chiral properties. These relationships are based on depolarization of waves reflected and transmitted through optically active media. Only the eight quasi off-diagonal elements of the Mueller matrix are sensitive to optical activity. () 2008 Society of Photo-Optical Instrumentation Engineers. [DOI: 10.1117/1.2979232]
\end{abstract}

Subject terms: polarimetric; scatterometer; Mueller matrix; cross sections; ellipsometry; optical activity.

Paper 080275R received Apr. 9, 2008; revised manuscript received Jul. 7, 2008; accepted for publication Jul. 22, 2008; published online Sep. 18, 2008.

\section{Introduction}

Measurements of the Mueller matrix have numerous civilian and defense applications. These measurements can be used to validate analytical solutions for remote sensing of random media, such as the earth and sea surfaces. It has the potential to be used for the purposes of detecting and identifying biological and chemical threat agents. Specific combinations of the Mueller matrix elements can be used to characterize the optical rotation and circular dichroism of optically active materials. Moreover, it is possible to measure the complex chiral parameter of materials with dissipative host media.

The optical scatterometer described in this paper can measure all 16 elements of the Mueller matrix for arbitrary incident and scatter angles in the total solid angle $4 \pi$ (which includes reflections and transmission). The

0091-3286/2008/\$25.00 @ 2008 SPIE versatility of the instrument is of special interest because it can be used to determine which specific combination of the Mueller matrix elements can most reliably characterize natural or artificial materials that are optically active. It can also be used to verify the optimal mode of operationreflection from or transmission through the material. It is known that optically active materials do not depolarize normally incident waves. Therefore, the Mueller matrix cannot be used to detect optical activity at normal incidence. Because the chiral parameter often used in the constitution relations to represent the optical activity of the material is very small compared to the wavelength, the scatterometer can be used to determine the optimum angles of incidence for purposes of identification and characterization of optically active material. The optical polarimetric scatterometer can also be used to make measurements that are traditionally used to measure the ellipsometric parameters as well as the like and cross-polarized scatter cross sections used in remote sensing. 


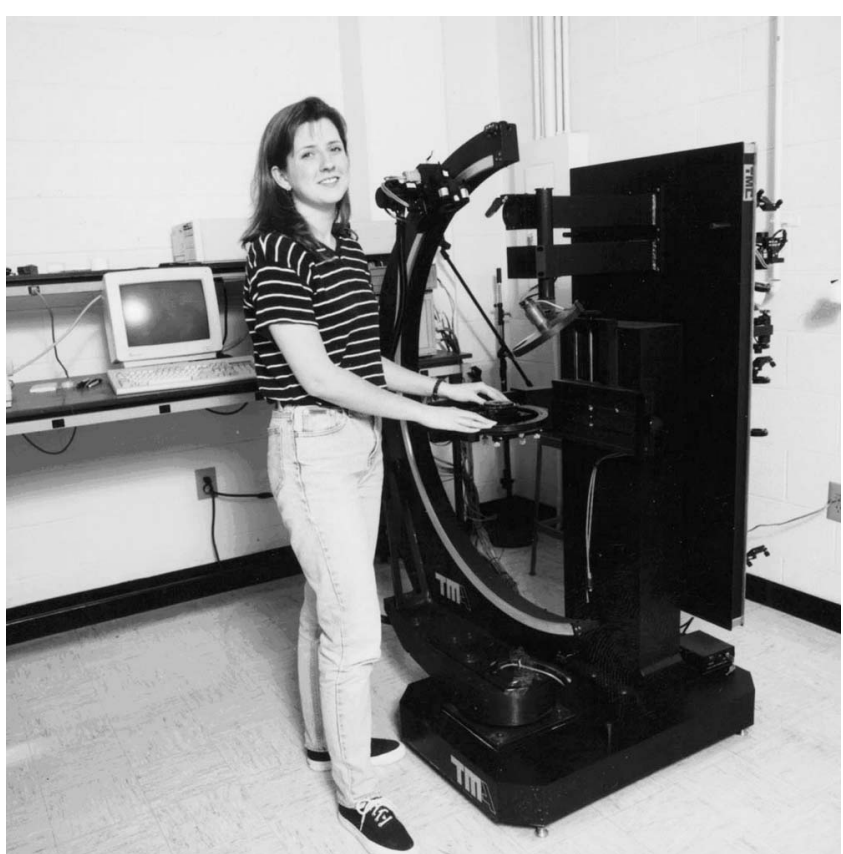

Fig. 1 View of the polarimetric scatterometer.

\section{Incident and Scattered Stokes Vectors and the Mueller Matrix}

The Stokes vector completely characterizes polarized light. ${ }^{1,2}$ The Mueller matrix relates the incident Stokes vector to the scattered Stokes vector. They are used to determine how light is modified upon interaction with material medium. For example, in ellipsometry ${ }^{3}$ only the relative intensity and the relative phase of the vertically and horizontally polarized specularly reflected light are measured and, in remote sensing, usually only those elements of the Mueller matrix that relate the vertically and horizontally polarized incident intensities to the scattered intensities are measured.

The TASC instrument (Schmidt Measurement Systems, Inc., Portland, Oregon) (Fig. 1) produces six incident Stokes vectors and measures six corresponding scattered (reflected/transmitted) Stokes vectors. Through combinations of these measurements all 16 elements of the Mueller matrix are determined. The redundancy in the measurements is used to minimize errors. One of the unique features of this instrument is that the receiver can be located in and out of the plane of incidence (defined by the incident electromagnetic wave vector and the normal to the mean surface of the sample). Thus, bistatic measurements can be made in the total solid angle $4 \pi$ (Fig. 2). Two coherent light sources at 0.6328 and $1.063 \mu \mathrm{m}$ are currently used in the operation of the scatterometer. The instrument can be retrofitted to include additional light sources.

\section{Components of the Scatterometer}

The system is designed such that the transmitter and receiver can rotate in arcs in vertical planes. The sample is at a distance $50 \mathrm{~cm}$ from the receiver. The normal to the mean plane of the sample is along the vertical axis. The sample holder can rotate the sample in the horizontal plane in order to vary the azimuth angle $\left(\phi^{i}\right)$ of the incident wave vector.

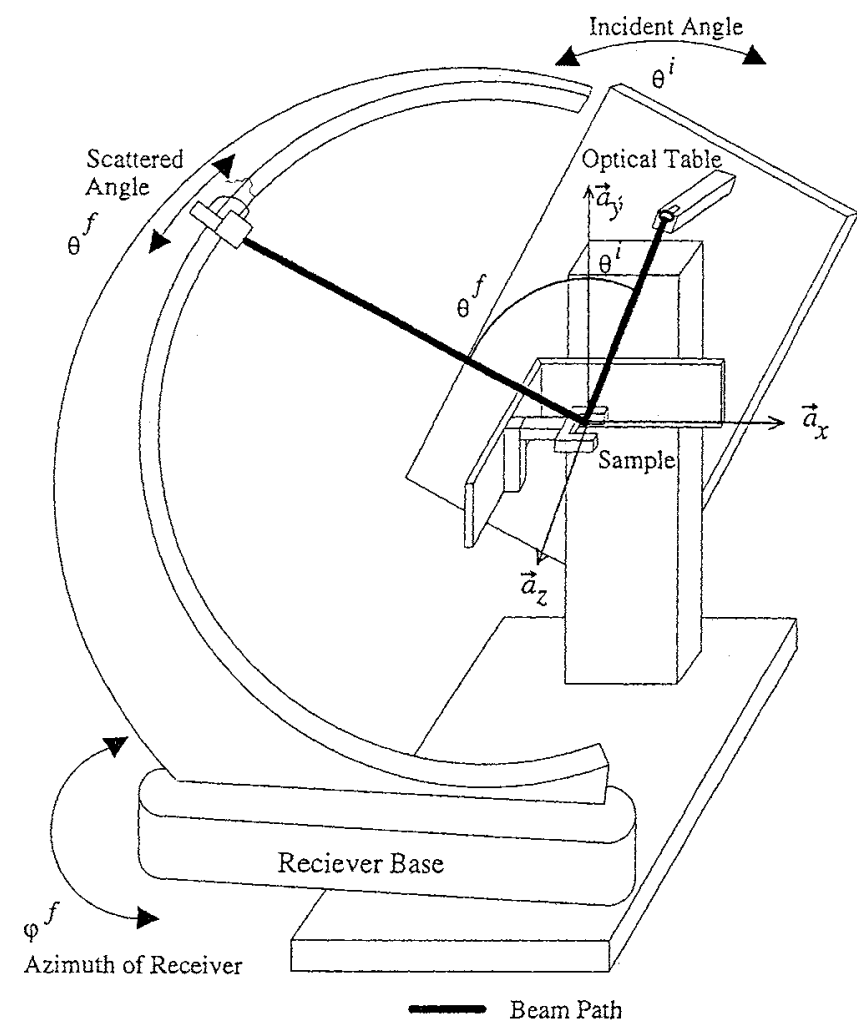

Fig. 2 The true angle scatter coordinate (TASC) system scatterometer, Schematic.

Variation of the incident elevation angle $\left(0 \leqslant \theta^{i}\right.$ $\leqslant 135 \mathrm{deg}$ ) measured from the vertical axis is achieved by rotating the optical table on which the sources are mounted (Fig. 2). The receiver located in the scatter direction can rotate in a 180-deg arc in vertical planes in order to vary the elevation angle $\left(\theta^{f}\right)$ of the scattered wave vector. The base of the receiver mount can rotate in a 180-deg arc in order to vary the azimuth angle of the scattered wave vector $\phi^{f}$. The laser sources are aligned such that the beams have a common path on exciting a beam combiner (Fig. 3). The system is currently excited by two laser sources (see Table 1).

On exiting the beam combiner, the laser beam encounters a calcite polarizer, which is used to set the initial polarization state of the beam before it enters the source polarization optics. The beam is chopped by an ac synchronous motor turning fanlike blades in order to detect the scattered signal. The beam is partially deflected to a reference detector in order to damp the laser power fluctuations. This reference signal is also used to synchronize the lock-in amplifier. On reflection from the combined beam turning mirror, the laser beam is directed toward the source polarization optics components. These components include a half-wave plate and a quarter-wave plate. They are mounted in rotary stages that are computer controlled. Each laser beam has its own set of source polarization optics. The source polarization optics produces six different incident wave polarization states: Vertical $(\mathrm{V}$, electric field parallel to the plane of incidence), Horizontal $(\mathrm{H}$, electric field perpendicular to the plane of incidence), right circular, left 


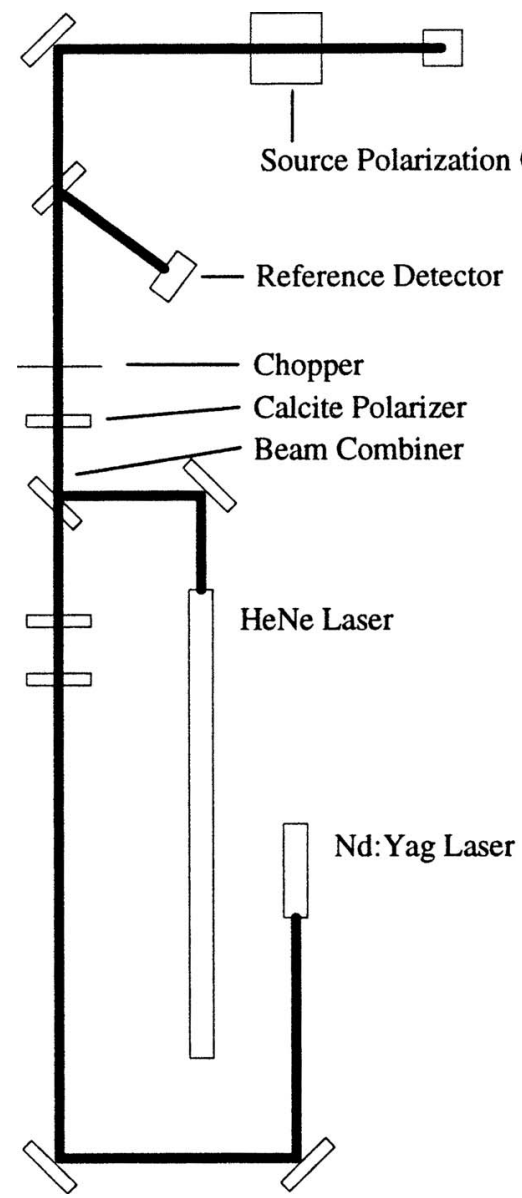

Fig. 3 Schematic of rear view of optical table containing the lasers and source optics.

circular, linear polarizations +45 or $-45 \mathrm{deg}$ with respect to the plane of incidence. After the desired polarization state is generated, the beam is passed through the optical table and down toward the sample (see Fig. 2).

The receiver assembly is mounted on the arm in the vertical plane. This assembly consists of a quarter-wave plate, a polarizer, a preamplifier, and a detector. The detectors for both wavelengths are made of silicon. They consist of filters and optical components that enhance its operation at the selected wavelength. The receiver polarizer and quarter-wave plate are mounted in rotary stages that are similar to those of the source polarization optics. The receiver polarizations (same as the six polarization states for

Table 1 TASC optical sources.

\begin{tabular}{lcc}
\hline \hline & Source 1 & Source 2 \\
\hline Laser type & HeNe & Nd:Yag \\
Wavelength $(\mu \mathrm{m})$ & 0.6328 & 1.063 \\
Power $(\mathrm{mW})(\mathrm{CW})$ & 7 & 10 \\
\hline \hline
\end{tabular}
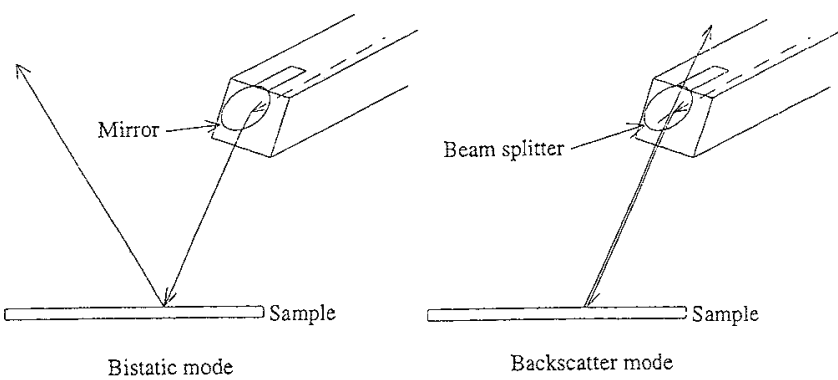

Fig. 4 Bistatic and backscatter modes of operation.

the transmitter) is computer controlled. The whole system is controlled by a central software package. The motorized stages in this equipment are as follows: ${ }^{4}$

1. Source components Half-wave plate, quarter-wave plate, and incident elevation angle

2. Receiver components: Quarter-wave plate, half-wave plate, receiver elevation angle, and receiver azimuth plane

3. Sample components: The mean plane of the sample can be rotated in order to vary the azimuth angle $\phi^{i}$. Optional computer-operated components consist of sample $x y$ (horizontal) and $z$ (vertical) translations, beam expansion, beam focus, and sample rotation. The instrument has the ability to operate in the retro (backscatter) mode by replacing the mirror that directs the laser beam down to the sample by a beamsplitter (see Fig. 4). The backscatter mode is the mode of operation most commonly associated with active remote sensing.

\section{Stokes Vectors and Mueller Matrices}

A polarized electromagnetic wave can be fully characterized by the Stokes vectors. ${ }^{5}$ The Stokes vector is given by

$$
\begin{aligned}
\mathbf{I}^{j} & =\left[\begin{array}{c}
I \\
M \\
C \\
S
\end{array}\right]=\left[\begin{array}{l}
\rho\left\{E_{x} E_{x}^{*}\right\}+\rho\left\{E_{y} E_{y}^{*}\right\} \\
\rho\left\{E_{x} E_{x}^{*}\right\}-\rho\left\{E_{y} E_{y}^{*}\right\} \\
\rho\left\{E_{x} E_{y}^{*}\right\}+\rho\left\{E_{x}^{*} E_{y}\right\} \\
i\left(\rho\left\{E_{x}^{*} E_{y}\right\}-\rho\left\{E_{x} E_{y}^{*}\right\}\right)
\end{array}\right] \\
& =\left[\begin{array}{l}
\rho\left\{E_{x} E_{x}^{*}\right\}+\rho\left\{E_{y} E_{y}^{*}\right\} \\
\rho\left\{E_{x} E_{x}^{*}\right\}-\rho\left\{E_{y} E_{y}^{*}\right\} \\
2 \operatorname{Re}\left(\rho\left\{E_{x} E_{y}^{*}\right\}\right) \\
2 \operatorname{Im}\left(\rho\left\{E_{x} E_{y}^{*}\right\}\right)
\end{array}\right] .
\end{aligned}
$$

In (1) $\rho\{\cdot\}$ denotes average, the asterisk denotes complex conjugate, and $\operatorname{Re}\{\cdot\}$ and $\operatorname{Im}\{\cdot\}$ denote real and imaginary parts, respectively. The Stokes vector for waves scattered above the specimen $I^{S}$ is related to the incident Stokes vector $I^{i}$ by the $(4 \times 4)$ Mueller matrix. For convenience, it is expressed as follows in terms of four $(2 \times 2)$ matrices:

$\mathbf{M}=\left[\begin{array}{l}M^{\mathrm{TL}} M^{\mathrm{TR}} \\ M^{\mathrm{BL}} M^{\mathrm{BR}}\end{array}\right]$, 
$\mathbf{M}^{\mathrm{TL}}=\left[\begin{array}{ll}\frac{1}{2}\left(\rho\left\{D^{\mathrm{HH}} D^{\mathrm{HH}}\right\}+\rho\left\{D^{\mathrm{HV}} D^{\mathrm{HV}}\right\}+\rho\left\{D^{\mathrm{VH}} D^{\mathrm{VH}}\right\}+\rho\left\{D^{\mathrm{VV}} D^{\mathrm{VV}}\right\}\right) & \frac{1}{2}\left(\rho\left\{D^{\mathrm{HH}} D^{\mathrm{HH}}\right\}-\rho\left\{D^{\mathrm{HV}} D^{\mathrm{HV}}\right\}+\rho\left\{D^{\mathrm{VH}} D^{\mathrm{VH}}\right\}-\rho\left\{D^{\mathrm{VV}} D^{\mathrm{VV}}\right\}\right) \\ \frac{1}{2}\left(\rho\left\{D^{\mathrm{HH}} D^{\mathrm{HH}}\right\}+\rho\left\{D^{\mathrm{HV}} D^{\mathrm{HV}}\right\}-\rho\left\{D^{\mathrm{VH}} D^{\mathrm{VH}}\right\}-\rho\left\{D^{\mathrm{VV}} D^{\mathrm{VV}}\right\}\right) & \frac{1}{2}\left(\rho\left\{D^{\mathrm{HH}} D^{\mathrm{HH}}\right\}-\rho\left\{D^{\mathrm{HV}} D^{\mathrm{HV}}\right\}-\rho\left\{D^{\mathrm{VH}} D^{\mathrm{VH}}\right\}+\rho\left\{D^{\mathrm{VV}} D^{\mathrm{VV}}\right\}\right)\end{array}\right]$,

$\mathbf{M}^{\mathrm{BL}}=\left[\begin{array}{cc}\operatorname{Re}\left(\rho\left\{D^{\mathrm{HH}} D^{\mathrm{VH}}\right\}+\rho\left\{D^{\mathrm{VV}} D^{\mathrm{HV}}\right\}\right) & \operatorname{Re}\left(\rho\left\{D^{\mathrm{HH}} D^{\mathrm{VH}}\right\}-\rho\left\{D^{\mathrm{VV}} D^{H V^{*}}\right\}\right) \\ -\operatorname{Im}\left(\rho\left\{D^{\mathrm{HH}} D^{\mathrm{VH}^{*}}\right\}-\rho\left\{D^{\mathrm{VV}} D^{\mathrm{HV}}\right\}\right) & -\operatorname{Im}\left(\rho\left\{D^{\mathrm{HH}} D^{\mathrm{VH}^{*}}\right\}+\rho\left\{D^{\mathrm{VV}} D^{\mathrm{HV}^{*}}\right\}\right)\end{array}\right]$,

$\mathbf{M}^{\mathrm{TR}}=\left[\begin{array}{cc}\operatorname{Re}\left(\rho\left\{D^{\mathrm{HH}} D^{\mathrm{HV}}\right\}+\rho\left\{D^{\mathrm{VV}} D^{\mathrm{VH}}\right\}\right) & \operatorname{Im}\left(\rho\left\{D^{\mathrm{HH}} D^{\mathrm{HV}}\right\}-\rho\left\{D^{\mathrm{VV}} D^{\mathrm{VH}}\right\}\right) \\ \operatorname{Re}\left(\rho\left\{D^{\mathrm{HH}} D^{\mathrm{HV}^{*}}\right\}-\rho\left\{D^{\mathrm{VV}} D^{\mathrm{VH}^{*}}\right\}\right) & \operatorname{Im}\left(\rho\left\{D^{\mathrm{HH}} D^{\mathrm{HV}^{*}}\right\}+\rho\left\{D^{\mathrm{VV}} D^{\mathrm{VH}^{*}}\right\}\right)\end{array}\right]$,

$\mathbf{M}^{\mathrm{BR}}=\left[\begin{array}{cc}\operatorname{Re}\left(\rho\left\{D^{\mathrm{VV}} D^{\mathrm{HH}}\right\}+\rho\left\{D^{\mathrm{VH}} D^{\mathrm{HV}}\right\}\right) & \operatorname{Im}\left(-\rho\left\{D^{\mathrm{VV}} D^{\mathrm{HH}^{*}}\right\}+\rho\left\{D^{\mathrm{VH}} D^{\mathrm{HV}}\right\}\right) \\ \operatorname{Im}\left(\rho\left\{D^{\mathrm{VV}} D^{\mathrm{HH}^{*}}\right\}+\rho\left\{D^{\mathrm{VH}} D^{\mathrm{HV}}\right\}\right) & \operatorname{Re}\left(\rho\left\{D^{\mathrm{VV}} D^{\mathrm{HH}^{*}}\right\}-\rho\left\{D^{\mathrm{VH}} D^{\mathrm{HV}^{*}}\right\}\right)\end{array}\right]$.

The Mueller matrix elements given in the diagonal matrices $M^{\mathrm{TL}}$ and $M^{\mathrm{BR}}$ are related to the scatter cross sections and the ellipsometric parameters, and the off diagonal matrices $M^{\mathrm{TR}}$ and $M^{\mathrm{BL}}$ are related to the optical activity (optical rotation and circular dichroism) and the complex chiral parameter.

\subsection{Ellipsometric Parameters}

Because the Mueller matrix contains complete polarimetric information about the intensities and the relative phases of the scattered light, it has very general applications. In ellipsometry, ${ }^{3}$ the measurement that is of particular interest is the complex ratio of the vertically and horizontally polarized reflection coefficients $R_{\mathrm{VV}}$ and $R_{\mathrm{HH}}$ for specularly reflected light. This ratio (the so-called ellipsometric function) is commonly expressed as

$R=\frac{R_{\mathrm{VV}}}{R_{\mathrm{HH}}}=\left|\frac{R_{\mathrm{VV}}}{R_{\mathrm{HH}}}\right| \exp \left[j\left(\phi_{\mathrm{VV}}-\phi_{\mathrm{HH}}\right)\right]=\tan (\psi) \exp (j \Delta)$.

In (7), $\psi$ and $\Delta$ are known as the ellipsometric angles or ellipsometric parameters. Following Azzam et al., ${ }^{6}$ assuming that the surface is perfectly flat and no depolarization occurs, the elements of the Mueller matrix satisfy the special relationships $m_{11}=m_{22}, \quad m_{12}=m_{21}, \quad m_{33}=m_{44}$, and $m_{34}=-m_{43}$. In this case, the ellipsometric angles can be obtained uniquely from the Mueller matrix elements

$\psi=\frac{1}{2} \arccos \left(\frac{m_{12}}{m_{11}}\right)$

$\Delta=\arctan \left(\frac{m_{43}}{m_{33}}\right)$.

Care is taken in interpreting Eqs. (8) and (9) so that $\psi$ and $\Delta$ are in the correct quadrant.
In general, the assumption that the scattered light is not depolarized by the surface is not strictly satisfied. For this case, the ellipsometric parameters are extracted from the Mueller matrix elements using

$\psi=\frac{1}{2} \arccos \left(\frac{m_{12}+m_{21}}{m_{11}+m_{22}}\right)$,

$\Delta=\arctan \left(\frac{m_{43}-m_{34}}{m_{33}+m_{44}}\right)$.

As above, care should be taken in interpreting Eqs. (10) and (11) to obtain $\psi$ and $\Delta$ in the correct quadrant.

\subsection{Scatter Cross Sections}

In remote sensing applications, the most commonly measured quantities are the scatter cross sections; thus, it is useful to describe scattering from a random rough surface in terms of the modified Mueller matrix. ${ }^{7,8}$ The upper left $2 \times 2$ submatrix of the modified Mueller matrix contains the like and cross-polarized scattering cross sections. The modified Stokes vector is expressed as

$I_{m}^{j}=\left[\begin{array}{c}I_{x} \\ I_{y} \\ C \\ S\end{array}\right]=\left[\begin{array}{c}\rho\left\{E_{x} E_{x}^{*}\right\} \\ \rho\left\{E_{y} E_{y}^{*}\right\} \\ \rho\left\{E_{x} E_{y}^{*}\right\}+\rho\left\{E_{x}^{*} E_{y}\right\} \\ i\left(\rho\left\{E_{x}^{*} E_{y}\right\}-\rho\left\{E_{x} E_{y}^{*}\right\}\right.\end{array}\right]=\left[\begin{array}{c}\rho\left\{E_{x} E_{x}^{*}\right\} \\ \rho\left\{E_{y} E_{y}^{*}\right\} \\ 2 \operatorname{Re}\left(\rho\left\{E_{x} E_{y}^{*}\right\}\right) \\ 2 \operatorname{Im}\left(\rho\left\{E_{x} E_{y}^{*}\right\}\right)\end{array}\right]$

The corresponding modified Mueller matrix elements associated with $M_{m}^{\mathrm{TL}}$ are the like and cross-polarized cross sections. 


$$
\mathbf{M}_{m}^{\mathrm{TL}}=\left[\begin{array}{cc}
\rho\left\{R^{\mathrm{HH}} R^{\mathrm{HH}}\right\} & \rho\left\{R^{\mathrm{HV}} R^{\mathrm{HV}^{*}}\right\} \\
\rho\left\{R^{\mathrm{VH}} R^{\mathrm{VH}}\right\} & \rho\left\{R^{\mathrm{VV}} R^{\mathrm{VV}}\right\}
\end{array}\right]=\left[\begin{array}{cc}
\sigma^{\mathrm{HH}} & \sigma^{\mathrm{HV}} \\
\sigma^{\mathrm{VH}} & \sigma^{\mathrm{VV}}
\end{array}\right] .
$$

The cross sections (bidirectional reflective distribution functions) can also be measured directly by the scatterometer.

\subsection{Complex Chiral Parameters and Optical Activity (Optical Rotation and Circular Dichroism)}

Optical activity of biological and chemical materials is characterized by optical rotation and circular dichroism. ${ }^{9,10}$ The differences in the phase velocities of the two circularly polarized characteristic waves propagating across the material results in the rotation of the electric field of a linearly polarized incident wave. The differences in the attenuation constants for the two circularly polarized characteristic waves result in the ellipticity of the incident linearly polarized wave that propagates across the material. The ratio of the large to small axes of the ellipse characterizes the circular dichroism of the material.

Waves normally incident upon an optically active medium are not depolarized upon reflection and transmission, and the corresponding Mueller matrix elements are not impacted by the optical activity of the medium. At oblique angles of incidence, the optical activity of the medium does depolarize the reflected and transmitted waves. The eight quasi off-diagonal elements of the Mueller matrix are shown to be impacted by the optical activity of the medium (to first order in the chiral parameter in the constitutive relations that relate the electric and magnetic flux density vectors to the electric and magnetic fields). The relationship between the Mueller matrix elements and the optical activity is through the cross-polarized reflection (and transmissions) coefficients

$$
\begin{aligned}
R^{\mathrm{VH}} & =-R^{\mathrm{HV}}=T^{\mathrm{VH}}=-T^{\mathrm{HV}}\left(\frac{Z_{1}}{Z_{0}}\right) \\
& =\frac{1}{2} j k \beta T_{01}^{\mathrm{HH}} T_{10}^{\mathrm{VV}} \tan ^{2} \theta_{1} \\
& \equiv \frac{j k \beta f}{2} .
\end{aligned}
$$

In (14), the second superscript denotes the polarization of the incident wave $(\mathrm{P}=\mathrm{V}$ vertical and $\mathrm{P}=\mathrm{H}$ horizontal $)$. The first superscript denotes the polarization of the reflected or transmitted wave. The second subscript denotes the medium of the incident wave ( 0 , free space; 1 chiral medium) and the first subscript the medium of the transmitted wave, $k$ is the wavenumber of the host medium, $\beta$ is the complex chiral parameter, $Z_{i}$ denotes the characteristic impedance of the $i$ 'th medium, and $\theta_{1}$ is the angle of refraction in the host medium.

For a dissipative host medium (complex permittivity and permeability), the relationships between complex pairs of Mueller matrix elements and the cross-polarized coefficients (14) are given by

$m_{23}+j m_{41}=-m_{32}+j m_{14}=\left(R^{\mathrm{HH}}+R^{\mathrm{VV}}\right) R^{\mathrm{HV}}$

and $m_{13}+j m_{42}=-m_{31}+j m_{24}=\left(R^{\mathrm{HH}}-R^{\mathrm{VV}}\right) R^{\mathrm{HV}}$.

In (15) and (16), the asterisk denotes complex conjugate. Thus, on using the relationship between the complex chiral parameter $\beta$ and the optical activity, the specific optical rotation $\mathrm{OR}^{\prime}$ and circular dichroism, $\mathrm{CD}^{\prime}$ per unit length are given by

$\mathrm{OR}^{\prime}+j \mathrm{CD}^{\prime}=\frac{\frac{2 k\left(m_{41}+j m_{23}\right)}{\left(R^{\mathrm{HH}}+R^{\mathrm{VV}}\right)^{*}}}{f}=k^{2}\left(\beta^{\prime}+j \beta^{\prime \prime}\right)$

or

$\mathrm{OR}^{\prime}+j \mathrm{CD}^{\prime}=\frac{\frac{2 k\left(m_{24}+j m_{13}\right)}{\left(R^{\mathrm{HH}}-R^{\mathrm{VV}}\right)^{*}}}{f}=k^{2}\left(\beta^{\prime}+j \beta^{\prime \prime}\right)$

In (17) and (18), $f$ is as defined in (14). Similar expression can be written in terms of the other four quasi off-diagonal elements in (15) and (16). The optical polarimetric scatterometer is primary use related to the identification and characterization of optically active media, is for purposes of experimentally validating the analysis in order to determine the following:

1. optimum angle of incidence (14)

2. optimum mode of operation, reflection, or transmission

3. most suitable pair of Mueller matrix quasi offdiagonal elements for reliable and accurate measurements of the optical activity (17) and (18).

The Mueller matrix measurements for identifying and characterizing optically active material has the potential to be used for defense and homeland security by detecting biological and chemical threat agents and explosives. They also have the potential to be used in many medical, pharmacological, and clinical applications.

\subsection{Accuracy of Measurements of Mueller Matrix Elements}

In order to examine the accuracy of the measurements of the elements of the Mueller matrix over the full range of incident angles, a silicon wafer with a 100-nm layer of nickel was coated with a $100-n m$ layer of gold. The measured relative permittivity of the coated silicon wafer was $\varepsilon_{r}=11.94-j 1.65$ based on the measurement of the ellipsometric parameters. ${ }^{6}$ From measurements of the bidirectional reflectance distribution function, the root-meansquare (rms) height of slightly rough surfaces was $0.5 \mathrm{~nm} .{ }^{11}$ The surface height and slope probability density functions are assumed to be gamma functions of order $\kappa$ $=20$ (quasi Gaussian). ${ }^{12,13}$ Normalized Mueller matrix elements for the specularly reflected wave are plotted in Figs. 5(a)-5(e) together with predicted results based on the full wave theory. 14

In Figs. 5(a)-5(d), the normalized Mueller matrix elements $m_{22} / m_{11}, m_{33} / m_{11}, m_{34} / m_{11}, m_{43} / m_{11}$, and $m_{44} / m_{11}$ are plotted as functions of the angle of incidence. From all these plots, one finds that the largest errors occur for normal incidence, and they are less than 5\%. Because the sur- 


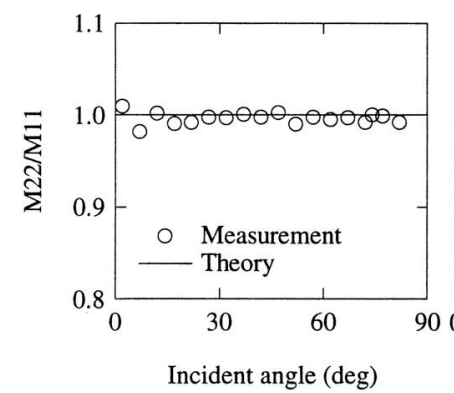

(a)

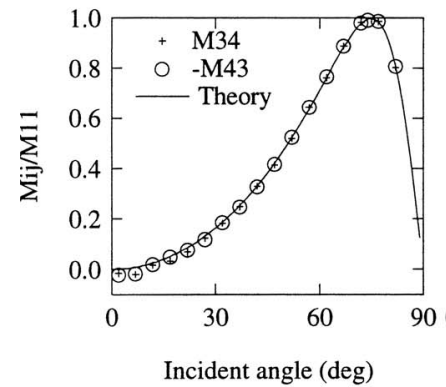

(c)

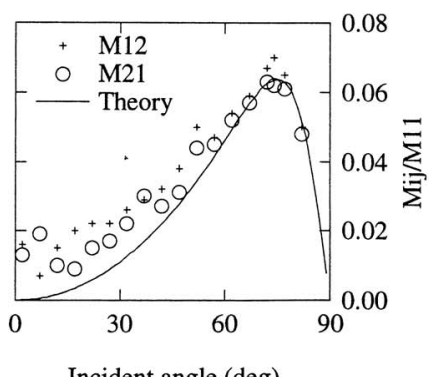

Incident angle (deg)

(e)

Fig. 5 Normalized Mueller matrix elements: (a) $m_{22} / m_{11}$, (b) $m_{33} / m_{11}$, (c) $m_{34} / m_{11}$ and $m_{43} / m_{11}$, (d) $m_{44} / m_{11}$, and (e) $m_{21} / m_{11}$ and $m_{12} / m_{11}$.

face is slightly rough, depolarization in the specular direction is negligible and $m_{33} \approx m_{44}$ and $m_{34} \approx m_{43}$.

The comparisons are quite different in Fig. 5(e) for the normalized Mueller matrix elements $m_{12} \approx m_{21}=\left|R^{\mathrm{HH}}\right|^{2}$ $-\left|R^{\mathrm{VV}}\right|^{2}$. For the Mueller matrix elements, the errors are significantly larger than for the four previous cases considered, particularly near normal incidence. The reason for this is that at normal incidence $\left|R^{\mathrm{HH}}\right|^{2}-\left|R^{\mathrm{VV}}\right|^{2}=0$ and the relative errors are significantly larger when the measurements involve differences between two near-equal numbers. The difference between $\left|R^{\mathrm{HH}}\right|$ and $\left|R^{\mathrm{VV}}\right|$ peaks at $\sim 70$ deg at a small value 0.06 , where the error is smaller than for normal incidence.

This observation has a significant impact on the choice of the specific pairs of Mueller matrix elements for purposes of measuring the optical activity (17) or (18). At near-normal incidence $R^{\mathrm{HH}} \approx-R^{\mathrm{VV}}$ and for near-grazing incidence $R^{\mathrm{HH}} \approx R^{\mathrm{VV}} \rightarrow-1$. It has also been shown that the function $f$ defined in (14) peaks for angles near-grazing incidence. ${ }^{15}$ Taken together, these observations make measurements based on (17) preferable to (18). Note also that because $R^{\mathrm{VH}}=-R^{\mathrm{HV}}, m_{44}=m_{14}$ and $m_{23}=-m_{32}$.

\section{Conclusion}

The optical polarimetric scatterometer described in this paper has numerous applications. It has been used to validate analytical solutions derived for electromagnetic wave scattering problems. Problems of scattering at frequencies far below the optical frequencies of the lasers that excite the scatterometer can also be considered by examining scaleddown laboratory models of the structures under consideration. ${ }^{16}$ The scatterometer not only measures all 16 elements of the Mueller matrix but also directly measures the four like and cross-polarized scatter cross sections (13). The ellipsometric parameters $\psi$ and $\Delta$ can also be measured in terms of the quasi-diagonal Mueller matrix elements for samples that have smooth or rough surfaces that depolarize the scattered waves $[(10),(11)]\}$. Furthermore, it has been shown recently ${ }^{17}$ that the optical activity (both optical rotation and circular dichroism) and the complex chiral parameters can be measured in terms of the quasi off-diagonal elements of the Mueller matrix [(17), (18)]. Thus measurements of all 16 elements of the Mueller matrix have specific applications. The elements of the $(2 \times 2)$ matrix $M^{\mathrm{TL}}$ are related to the scatter cross sections, (see Section 4.2) the elements of the $(2 \times 2)$ matrices $M^{\mathrm{TL}}$ and $M^{\mathrm{BR}}$ are related to the ellipsometric parameters (see Section 4.1), and the two off diagonal matrices $M^{\mathrm{BL}}$ and $M^{\mathrm{TR}}$ are related to optical activity (see Section 4.3). The applications include identification and characterization of chemical and biological materials, validation of remote sensing techniques, and monitoring thin-film technology. The polarimetric measurements are useful in biochemistry, biomedicine, bioengineering, and electro-optics. The measurements taken by the optical polarimetric scatterometer (in the total solid angle $4 \pi$ ) is computer controlled and can be taken rapidly. ${ }^{18}$ Considerations related to avoiding unnecessarily large errors in the measurements have also been considered so that they can be kept below $5 \%$ for most applications. For example, one concludes from the accuracy demonstrated by Fig. 5(e) that the measurements based on (17) are preferable to the measurements based on (18).

\section{Acknowledgments}

The authors are grateful for the encouragement and suggestions of Dr. A. Carrieri and Dr. A. Lakhtakia for the paper. The authors also thank Jane Craig, R. Odom and M. Lammers for preparing the paper, and Gabe Ives, for the artwork.

\section{References}

1. M. Born and E. Wolf, Principles of Optics, 3rd ed., pp. 30-32, 554 555, Pergamon Press, London (1965).

2. W. A. Shurcliff, Polarized Light, 1st ed., pp. 109-123, Harvard University Press, Cambridge, MA (1966).

3. R. M. A. Azzam and N. M. Bashara, Ellipsometry and Polarized Light, 1st ed., pp. 173-174, North-Holland, Amsterdam (1977).

4. TMA, TASC Polarimetric Scatterometer, Instrument operation manual, TMA Technologies, Inc., Bozeman, Montana (1992).

5. G. G. Stokes, "On the composition and resolution of streams of polarized light from different sources," Trans. Cambridge Philos. Soc. 9. 399-416 (1852)

6. R. M. A. Azzam, K. A. Guardian, and A. G. Lopez, "Conventional and generalized Mueller-matrix ellipsometry using the four-detector photo-polarimeter," Opt. Eng. 30(10), 1583-1589 (1991).

7. S. Chandrasekhar Radiative Transfer, Dover, New York (1950).

8. A. Ishimaru, Wave Propagation and Scattering in Random Media, Academic Press, New York (1978). 
9. L. Barron, Molecular Light Scattering and Optical Activity, 2nd ed., Cambridge University Press, Cambridge, England (2004).

10. A. Lakhtakia, "Beltrami Fields in Chiral Media," World Scientific in Contemporary Chemical Physics, Vol. 2, Singapore (1994).

11. J. C. Stover, Optical Scattering: Measurement and Analysis, McGraw-Hill, New York (1990).

12. P. Beckman, Orthogonal Polynomials for Engineers and Physicists, Golem Press, Boulder, CO (1968)

13. P. Beckman, "Scattering by non-Gaussian surfaces," IEEE Trans. Antennas Propag. AP-21(2), 169-175 (1973).

14. E. Bahar, "Review of the full wave solutions for rough surface scattering and depolarization: Comparison with geometric and physical optics, perturbation and two-scale hybrid solutions," J. Geophys. Res. 92(C5), 749-759 (1988).

15. E. Bahar, "Optimum electromagnetic wave excitations of complex media characterized by positive or negative refractive indices and by chiral properties," J. Opt. Soc. Am. B 24, 2807-2813 (2007).

16. E. Bahar, "Laboratory models used in the investigation of radio wave propagation in irregular structure," IEEE Trans. Antennas Propag. 48(9), 1-9 (2000).

17. E. Bahar, "The relationship between optical rotation and circular dichroism and elements of the Mueller matrix for natural and artificial chiral materials," J. Opt. Soc. Am. B 25(2), 218-222 (2008).

18. R. Kubik and E. Bahar, "Measurements of a polarimetric optical bistatic scatterometer," Proc. of Combined Optical Microwave Earth and Atmospheric Sensing (CO_MEAS' 93), pp. 173-176, IEEE, Piscataway, NJ (1993).

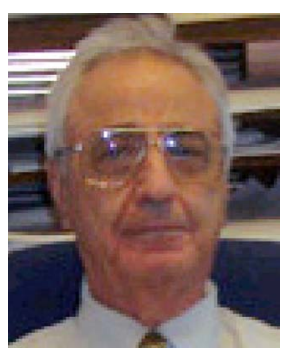

Ezekiel Bahar, a George Holmes Distinguished University Professor, received his BSc and MSc from Technion, Israel Institute of Technology in 1958 and 1960, respectively, and his $\mathrm{PhD}$ in 1964 from the University of Colorado. He began his teaching career at Technion and has been professor at the University of Colorado, University of $\mathrm{Ne}$ braska, Lincoln, visiting professor at the $\mathrm{Na}$ tional Oceanographic and Atmospheric Administration, and Durham professor at the University of Nebraska, where he also served as assistant vice chancellor for Academic Affairs, director of the Office of Academic Program Reviews and president of the Faculty Senate. Bahar received an award for outstanding research and creative activity from the University of Nebraska and an IEEE Fellow Award for contributions to the theory of propagation, scattering, and depolarization of electro-magnetic waves.

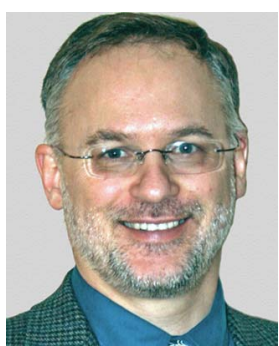

Robert D. Kubik received his BSEE in 1987, MSEE in 1989, and PhD in 1993 from the University of Nebraska. He is with Motorola's Global Government Affairs office in Washington DC, where he is director of Telecom Relations Global. He is currently vice-chairman of the Regulatory Group in the WiMAX Forum. Kubik is responsible for the development of regulatory policy in new spectrum management areas, including WiMAX, advanced wireless services, and the use of cognitive radio to access unused spectrum. He works with the FCC and other U.S. domestic regulators in spectrum policy and has played key roles in Motorola's efforts in defining regulatory policies regarding cellular, fixed/mobile broadband, mobile satellite, and the use of license-exempt spectrum. 